\title{
REVIEW
}

\section{Diagnosing cardiac contusion: old wisdom and new insights}

K C Sybrandy, M J M Cramer, C Burgersdiik

Heart 2003;89:485-489

Cardiac contusion is usually caused by blunt chest trauma and therefore is frequently suspected in patients involved in car or motorcycle accidents. The diagnosis of a myocardial contusion is difficult because of non-specific symptoms and the lack of an ideal test to detect myocardial damage. Cardiac contusion can cause life threatening arrhythmias and cardiac failure. Many diagnostic methods, such as ECG, biochemical cardiac markers, transthoracic and transoesophageal echocardiography, and radionuclide imaging studies, have been investigated to determine their use in predicting such complications. Recently, cardiac troponin I and T were found to be highly sensitive for myocardial injury. Troponin I and T have also proved to be useful in the stratification of patients at risk for complications. Nevertheless, diagnosis of a cardiac contusion and identification of patients at risk remain a challenge. In this review the current diagnostic tests will be discussed. Also, based on these diagnostic tests, a screening strategy containing data from the latest studies is presented, with the intention of detecting patients at risk.

See end of article for authors' affiliations

Correspondence to Dr MM Cramer, Heart Lung Centre Utrecht, University Medical Centre, Department of Non-invasive Cardiac Imaging. HP F02.35 1, PO 85500, 3508 GA Utrecht, Netherlands:

m.j.m.cramer@hli.azu.nl

Accepted

20 November 2002
T he exact incidence of a cardiac contusion in patients with blunt chest trauma is unknown. The reported incidence ranges between $3-56 \%$ of patients, depending on the criteria used for establishing the diagnosis. High incidences of cardiac injury, varying between 29\% and $56 \%$, were found in ECG studies. ${ }^{1-5}$ In studies of the cardiospecific $\mathrm{MB}$ isoenzyme of creatine kinase (CK-MB), incidences of approximately $19 \%$ were found. ${ }^{16}$ Echocardiographic evidence of myocardial injury was detected in 3-26\% of patients using a transthoracic approach ${ }^{1-3} 7-9$ and in $27-56 \%$ of the patients using a transoesophageal approach. ${ }^{9-12}$ Recent studies that used the highly specific cardiac markers troponin I or T for the diagnosis of a cardiac contusion reported incidences ranging between 15-24\%. ${ }^{58}$ Therefore, a substantial number of patients with blunt chest trauma are suspected of having cardiac injury.

Possible complications of cardiac injury, such as life threatening ventricular arrhythmias and cardiac failure, are less common and were described in up to $16 \%$ of patients. ${ }^{1-3913}{ }^{14}$ If patients receive adequate treatment for such complications, death due to cardiac causes is rare. In one study, one patient $(1.5 \%)$ died of persistent cardiogenic shock and in another study three severely injured patients died before treatment had been started. $^{28}$

In mildly injured patients a cardiac contusion is generally well tolerated. The majority of such patients never suffer any symptoms and proceed to an uncomplicated recovery with an excellent prognosis. However, life threatening ventricular arrhythmias and cardiac failure can also occur in these symptomless patients. It is therefore important to screen all patients with blunt chest trauma to identify those at risk for complications.

\section{CAUSES AND CLINICAL FEATURES OF BLUNT CHEST TRAUMA}

Blunt cardiac injury is most frequently caused by road traffic accidents and results from a direct blow to the chest from a steering wheel or rapid deceleration. ${ }^{26}{ }^{13} \mathrm{~A}$ direct blow to the precordium or a sustained force to the thoracic wall compresses the heart between the sternum and spine. In rapid deceleration accidents the heart, which has relatively free movement in the anteroposterior direction, continues to move forward due to its momentum and strikes the internal sternum with considerable force. Other less frequent causes are falls from a great height, sport injuries, blast forces, and indirect compression on the abdomen with upward displacement of abdominal viscera. ${ }^{13} 15$

Cardiac contusion clinically presents as a spectrum of injuries of varying severity ${ }^{6}{ }^{13}{ }^{16}$ Patients with severe myocardial injury are easily detected in case of haemodynamic instability. However, cardiac symptoms such as hypotension or hypoxia can be easily masked in trauma patients due to other severe injuries with blood loss or associated pulmonary, vascular, and neurological injuries. The diagnosis of cardiac contusion in trauma patients without evident clinical signs of myocardial injury is more difficult. Myocardial contusion may produce only mild symptoms, such as palpitations or precordial pain, which is often attributed to concomitant musculoskeletal injury. Although the majority of patients with blunt chest trauma have chest wall lesions, absence of thoracic lesions decreases the suspicion but never excludes cardiac injury. ${ }^{15}{ }^{17}$ Chest radiography and thoracic computed tomography provide no additional information for the diagnosis of cardiac

Abbreviations: $C K$, creatine kinase; $C K-M B$, cardiospecific $M B$ isoenzyme of creatine kinase; TOE, transoesophageal echocardiography; SPECT, single photon emission computed tomography 


\begin{tabular}{l} 
Table 1 Electrocardiographic findings in cardiac \\
contusion \\
\hline Non-specific abnormalities \\
Pericarditis-like ST segment elevation or PTa depression \\
Prolonged QT interval \\
Myocardial injury \\
New Q wave \\
ST-T segment elevation or depression \\
Conduction disorders \\
Right bundle branch block \\
Fascicular block \\
AV nodal conduction disorders (1, 2, and 3 degree AV block) \\
Arrhythmias \\
Sinus tachycardia \\
Atrial and ventricular extrasystoles \\
Atrial fibrillation \\
Ventricular tachycardia \\
Ventricular fibrillation \\
Sinus bradycardia \\
Atrial tachycardia \\
\hline AV, atrioventricular.
\end{tabular}

contusion but may show associated injury of the great vessels, or skeletal or pulmonary structures. ${ }^{18-20}$ The role of magnetic resonance imaging in the detection of acute myocardial injury has not yet been established..$^{18}$ However, for the evaluation of the spine, diaphragm, and thoracic aorta in haemodynamically stable patients with blunt chest trauma, magnetic resonance imaging has been described as an accurate alternative to computed tomography, in case of suboptimal images. ${ }^{18} 21$

\section{BIOCHEMICAL CARDIAC MARKERS}

A cardiac contusion is histologically characterised by intramyocardial haemorrhage, oedema, and necrosis of myocardial muscle cells. These histological findings are almost similar to those seen in acute myocardial infarction. ${ }^{1322}$ Therefore, cardiac enzyme determinations were the first screening tool used to detect myocardial injury. Creatine kinase (CK) is nonspecifically increased in trauma patients caused by associated skeletal muscle injury. CK-MB is known to have a better specificity in acute myocardial infarction. However, in multitrauma with high CK concentrations many false positive increases were found ${ }^{6}{ }^{13}$ The usefulness of CK-MB determination seemed to be restricted to the detection of myocardial trauma in case of mild non-cardiac injuries. ${ }^{23}$ More recent studies reported low sensitivity and specificity of CK-MB for cardiac injury in this category of mildly injured patients. ${ }^{3}{ }^{14} 24$ Therefore, CK-MB determination is of limited value in the detection of myocardial injury.

Serum cardiac troponins, troponin I and troponin $\mathrm{T}$, are highly specific to myocardial injury. They are myocardial regulatory contractile proteins not found in skeletal muscles and are released into the circulation only after loss of membrane integrity. The latest studies showed high accuracy of both troponin I and troponin $\mathrm{T}$ in the diagnosis of cardiac injury. ${ }^{78}$ Moreover, a normal concentration of cardiac troponin I or $\mathrm{T}$ has been reported by several investigators to be a strong indicator for the absence of cardiac injury in patients with blunt chest trauma. ${ }^{3578}$

To diagnose a cardiac contusion, the ideal timing of blood sampling for troponin assays has not yet been determined. Some investigators found that both troponin I and troponin $\mathrm{T}$ can reach peak values earlier following cardiac trauma than after acute myocardial infarction. ${ }^{36}$ However, if troponin I or $\mathrm{T}$ concentrations are within reference ranges on admission shortly after trauma, a secondary measurement after 4-6 hours is necessary to reliably exclude myocardial injury. ${ }^{26}$ Increased troponin I or T may persist for 4-6 days and thus
Table 2 Echocardiographic findings in acute cardiac contusion

Transthoracic echocardiography

Regional wall motion abnormalities

Pericardial effusion

Valvar lesions

Right and left ventricular enlargement

Ventricular septum rupture

Intracardiac thrombus

Transoesophageal echocardiography

Aortic endothelial laceration or aortic dissection

Aortic rupture

$$
\text { Aortic rupture }
$$

may also facilitate an evaluation for cardiac damage of patients presenting days after the injury. ${ }^{3}$

\section{ELECTROCARDIOGRAPHY}

The ECG after blunt chest trauma may be normal or may show non-specific abnormalities (table 1)..$^{2-5}{ }^{12-15} 17$ Because of its anterior position in the thorax and proximity to the sternum, the right ventricle is far more frequently injured than the left ventricle. ${ }^{1679172027}$ The ECG mainly reflects the electrical activity of the left ventricle because of its greater mass. The ECG is relatively insensitive to right ventricular electrical activity. For that reason, a cardiac contusion usually results in moderate right ventricular damage with only minor electrical changes, which can easily be missed on an ECG. Disappointingly, in a study by Walsh and colleagues, ${ }^{28}$ patients with and without blunt chest trauma could not be differentiated by right precordial lead recording (V4R). ${ }^{28}$

Non-specific ECG abnormalities are often seen in trauma patients. Some of these ECG changes may be caused by noncardiac factors such as hypoxia, anaemia, abnormal concentrations of serum electrolytes, and changes in vagal or sympathetic tone.

Left ventricular injury can produce ST segment or T wave abnormalities or, in the case of extensive necrosis, new Q waves. ${ }^{413}$ Some ECG changes that require treatment appear only after several hours. So, at least one repeat ECG is recommended. ${ }^{13}{ }^{14}$

A severely injured right ventricle may cause a right bundle branch block, usually transient. ${ }^{13}{ }^{17}$ Less commonly, various degrees of atrioventricular block have been documented after blunt chest trauma. ${ }^{1}{ }^{13}{ }^{17}$ In addition, myocardial cell damage produces electrical instability, which may result in a variety of supraventricular or ventricular arrhythmias. ${ }^{4613}$ Sinus tachycardia and ventricular and atrial extrasystoles are the most frequently reported rhythm disorders. More serious arrhythmias such as atrial fibrillation, ventricular tachycardia, and ventricular fibrillation occur less often but may acutely compromise the haemodynamic state or even result in sudden death. ${ }^{4}$

\section{ECHOCARDIOGRAPHY}

Contused myocardial tissue not only resembles infarcted myocardial tissue histologically but also functionally. ${ }^{13}$ A myocardial contusion can be recognised by localised myocardial wall dysfunction. Consequently, two dimensional echocardiography, which provides a direct view of wall motion abnormalities, has been shown in several studies to be an excellent tool in the detection of cardiac injury (table 2). ${ }^{12} 927$ In addition, echocardiography may show associated valvar lesions, intracardiac shunts or thrombosis, pericardial effusion or tamponade, and ventricular dilatation. ${ }^{126122729}$ From a practical standpoint, other important advantages of echocardiography are its non-invasive nature and its ease of use at the bedside and in the emergency department. However, in 
Table 3 Early and late complications of cardiac contusion

\section{Early complications}

Ventricular and supraventricular arrhythmias

Myocardial wall rupture with haemopericardium and tamponade

Ventricular septum rupture

Valvar insufficiencies caused by valvar lesions and papillary muscle ruptures

Intracardiac thrombus resulting in thromboembolic events

Coronary artery lesions with acute myocardial infarction

Late complications

Ventricular aneurysm

Chronic dilated cardiac dysfunction

Cardiac dysfunction caused by structural cardiac lesions

Constrictive pericarditis

Ventricular arrhythmias originating from myocardial scar tissue or aneurysm

patients with painful chest wall injuries transthoracic echocardiographic access may be limited. In these patients transoesophageal echocardiography (TOE) can be performed. ${ }^{11}{ }^{12} 29$ Although TOE is risky because of the insertion of the probe in to the oesophagus and the frequent need for sedation of the conscious patient, it has been shown to be safe in the evaluation of patients who have suffered blunt chest trauma. Two studies reported no complications respectively in 68 and 117 TOE procedures performed on severely injured trauma patients. ${ }^{11}{ }^{12}$ Another study in critically ill intensive care patients described worsening of haemodynamics and respiratory difficulties with TOE in two patients (4\%) who had heart failure..$^{30} \mathrm{TOE}$ is also recommended in case of suspected lesions to the great vessels or if the transthoracic images are suboptimal. Standard two dimensional echocardiography cannot detect minor injury with only myocardial oedema but no wall motion abnormalities. This entity, often referred to as cardiac "concussion", however, can possibly induce life threatening arrhythmias. ${ }^{46}$

\section{RADIONUCLIDE IMAGING} Ventriculography

Because of the increased availability of echocardiography, the use of radionuclide ventriculography to detect wall motion abnormalities and determine ejection fractions has been largely abandoned..$^{15} 173132$

\section{Myocardial perfusion scintigraphy}

In the past, the ability of myocardial perfusion scintigraphy to detect perfusion defects was investigated for its value in the diagnosis of a cardiac contusion. ${ }^{33-38}$ The initial planar $99 \mathrm{~m}-\mathrm{Tc}$ pyrophosphate imaging studies were reported to have low sensitivity for cardiac injury because these scans showed only large transmural defects. ${ }^{37}{ }^{38}$ Later studies using 201 -thallium single photon emission computed tomography (SPECT) were more precise in the detection and localisation of small transmural and non-transmural defects of the left ventricular wall. ${ }^{33-36}$ However, because the right ventricle is poorly visualised with 201-thallium SPECT, an isolated right ventricular contusion can theoretically be missed. The value of myocardial perfusion scintigraphy in the setting of an acute cardiac contusion therefore remains limited.

\section{Positron emission tomography}

Positron emission tomography has been shown to have great advantages in the assessment of myocardial viability in general. However, no experience with positron emission tomography in patients with blunt chest trauma has been reported in the literature. ${ }^{39}$

\section{IDENTIFICATION OF PATIENTS AT RISK}

None of the older diagnostic tests mentioned above were found to be accurate enough to confirm a cardiac contusion or to be

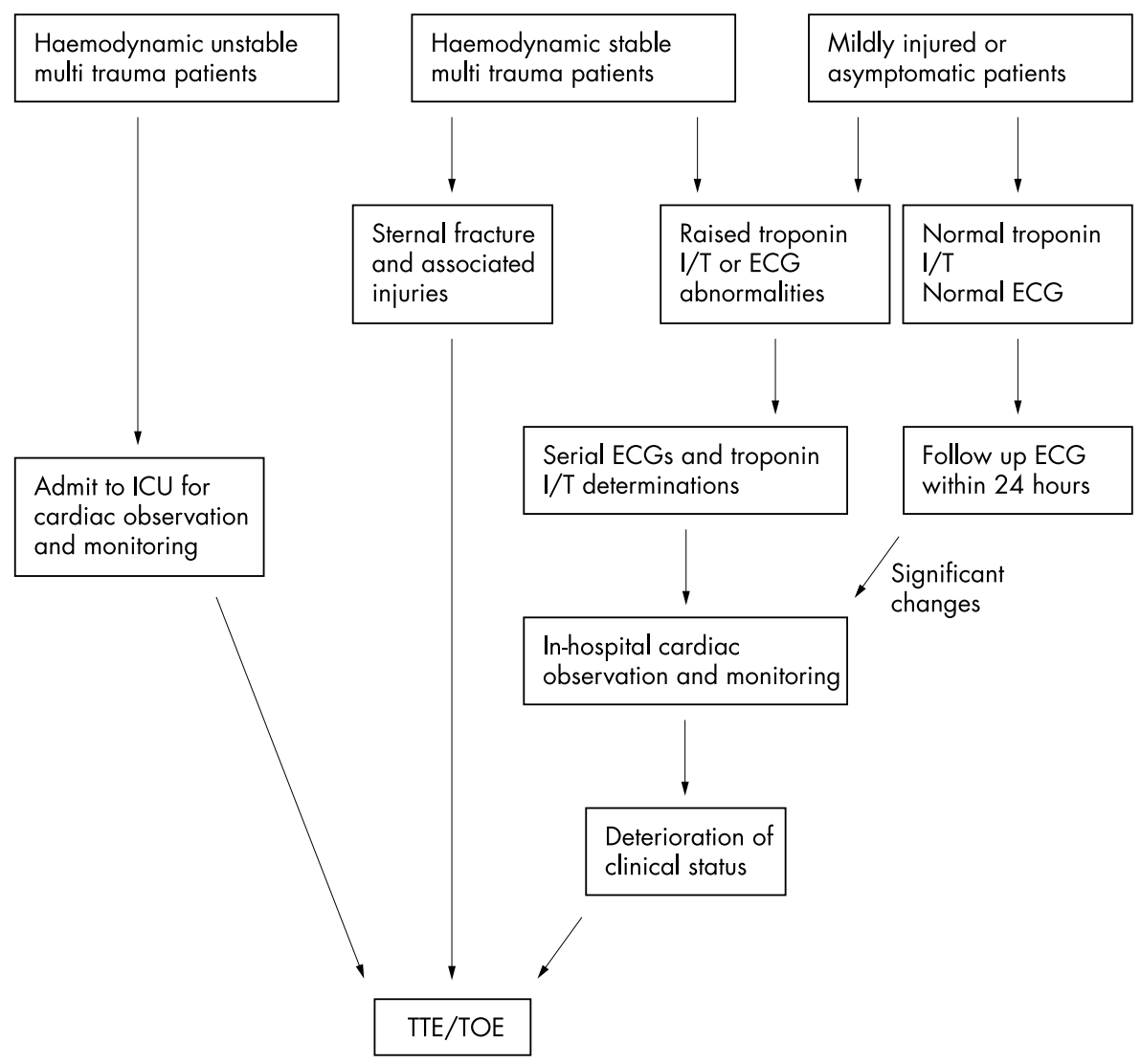

Figure 1 Screening strategy to identify patients at risk for cardiac complications in relation to the severity of associated injuries. ICU, intensive care unit; $\mathrm{TOE}$ transoesophageal echocardiography; TTE, transthoracic echocardiography.

TTE/TOE 
precise enough to predict all complications (table 3). However, the latest studies show that troponin I and troponin T are very helpful in the stratification of patients at risk for such complications. ${ }^{358}$ On the basis of these new data we suggest the following screening strategy, which is also illustrated in fig 1 .

Firstly, severely injured patients who present with haemodynamic instability must be suspected of having structural damage to the heart, the great vessels, or both and should have an echocardiographic examination as soon as possible. ${ }^{3691112}$

Secondly, for haemodynamically stable patients with multiple trauma, it is important to note that an isolated sternal fracture is not necessarily a sign of cardiac injury. ${ }^{20}$ However, in severely injured patients a sternal fracture may indicate cardiac injury and echocardiographic evaluation is recommended in these patients. ${ }^{41}{ }^{42}$ A small pericardial effusion associated with a sternal fracture, which is probably caused by pericardial irritation, neither implies cardiac injury nor increases the risk of tamponade. ${ }^{41}{ }^{42}$ Increased concentrations of cardiac troponin I and troponin T or ECG abnormalities indicate a higher risk of developing cardiac complications. Further investigation including echocardiography, serial ECGs, and troponin I determinations is advised. ${ }^{3} 812$

In a third group, consisting of mildly injured and asymptomatic patients, increased concentrations of CK-MB, troponin I or $\mathrm{T}$, and minor ECG abnormalities do not necessarily indicate a clinically significant cardiac contusion because not all these patients will develop complications. ${ }^{358142425}$ On the other hand, a normal concentration of cardiac troponin I or T rules out cardiac damage and consequently minimises the risk of cardiac complications. ${ }^{38}$ Therefore, in-hospital monitoring may be limited to patients with increased troponin concentrations or with significant ECG abnormalities. Serial ECGs and troponin determinations are advised until the results turn normal. $^{35}$ Echocardiography has rarely shown clinically significant functional or structural cardiac injuries in this group of patients. ${ }^{1241427}$ Echocardiography is very useful if the patient's clinical status deteriorates or if there is a discrepancy between clinical status, ECG, and cardiac enzyme concentrations. ${ }^{14}$

\section{TREATMENT OF CARDIAC INJURY}

If in-hospital cardiac monitoring is necessary, the hospitalisation period can be limited because nearly all (81-95\%) life threatening ventricular arrhythmias and acute cardiac failures occur within 24-48 hours after the trauma. ${ }^{5}{ }^{14}$

Treatment of a cardiac contusion in severely injured patients consists of haemodynamic stabilisation and treatment of associated injuries. In case early surgery is required general anaesthesia can be safely used. Within the first month following injury strict intraoperative haemodynamic monitoring is advisable. ${ }^{12461443}$

\section{PROGNOSIS}

Prognosis of patients in whom a cardiac contusion is diagnosed following a blunt chest trauma has been reported to be favourable. Severe cardiac injury with myocardial necrosis heals by scar formation, similar to that in ischaemic myocardial infarction. This may lead to ventricular aneurysm formation and in some cases to chronic heart failure. ${ }^{6445} \mathrm{~A}$ previous cardiac contusion must be taken into consideration in case of unexplained dilated cardiac dysfunction (especially if the right ventricle is affected), aneurysms of the ventricles or coronary arteries, unexplained constrictive pericarditis, or valvar lesions. ${ }^{16} 2244-46$

\section{CONCLUSION}

Diagnosing a cardiac contusion remains a significant challenge. Previously, none of the available diagnostic tests were sensitive or specific enough to determine cardiac injury. Nowadays, however, with the widespread availability of the cardiospecific troponin I and troponin $\mathrm{T}$ assays it has become much easier to detect myocardial injury, especially in the category of patients without evident signs of cardiac injury. Troponin I and troponin T have also facilitated the stratification of patients at risk for life threatening complications. A troponin I or T assay in combination with an ECG was shown to be sufficient for identifying the vast majority of patients who were at risk, thus avoiding extensive diagnostic screening. Moreover, patients with normal troponin I or T concentrations and ECG can be safely sent home. On the basis of these new data, we have proposed a screening protocol with different diagnostic strategies according to the severity of associated non-cardiac injuries.

\section{Authors' affiliations}

K C Sybrandy, M J M Cramer, Heart Lung Centre Utrecht, Department of Cardiology, University Medical Centre, Heidelberglaan 100, 3584 CX Utrecht, Netherlands

C Burgersdijk, Department of Cardiology, Medical Centre Alkmaar, Wilhelminalaan 12, 1815 JD Alkmaar, Netherlands

\section{REFERENCES}

1 Helling TS, Duke P, Beggs CW, et al. A prospective evaluation of 68 patients suffering blunt chest trauma for evidence of cardiac injury. J Trauma 1989:29:961-6.

2 Hiatt JS, Yeatman LA, Child JS. The value of echocardiography in blunt chest trauma. J Trauma 1988;28:914-22.

3 Bertinchant JP, Polge A, Mohty D, et al. Evaluation of incidence, clinical significance, and prognostic value of circulating cardiac troponin I and T elevation in hemodynamically stable patients with suspected myocardial contusion after blunt chest trauma. J Trauma 2000;48:924-31.

4 Illig KA, Swierzewski M, Feliciano DV, et al. A rational screening and treatment strategy based on the electrocardiogram alone for suspected cardiac contusion. Am J Surg 1991;162:537-44.

5 Salim A, Velmahos GC, Jindal A, et al. Clinically significant blunt cardiac trauma: role of serum troponin levels combined with electrocardiographic findings. J Trauma 2001;50:237-43.

6 Frazee RC, Mucha P Jr, Farnell MB, et al. Objective evaluation of blunt cardiac trauma. J Trauma 1986;26:510-20.

7 Adams JE III, Davila-Roman VG, Bessey PQ, et al. Improved detection of cardiac contusion with cardiac troponin I. Am Heart J 1996; 131:308-12

8 Collins JN, Cole FJ, Weireter L, et al. The usefulness of serum troponin levels in evaluating cardiac injury. Am Surg 2001;67:821-6.

9 Karalis DG, Victor MF, Davis GA, et al. The role of echocardiography in blunt chest trauma: a transthoracic and transesophageal echocardiographic study. J Trauma 1994;36:53-8.

10 Weiss RL, Brier JA, O'Connor W, et al. The usefulness of transesophageal echocardiography in diagnosing cardiac contusions. Chest 1996;109:73-7.

11 Brooks SW, Young JC, Cmolik B, et al. The use of transesophageal echocardiography in the evaluation of chest trauma. J Trauma 1992;32:761-8.

12 Garcia-Fernandez MA, Lopez-Perez JM, Perez-Castellano N, et al. Role of transesophageal echocardiography in the assessment of patients with blunt chest trauma: correlation of echocardiographic findings with the electrocardiogram and creatine kinase monoclonal antibody measurements. Am Heart J 1998;135:476-81.

13 Tenzer ML. The spectrum of myocardial contusion: a review. J Trauma 1985;25:620-7

14 Nagy KK, Krosner SM, Roberts RR, et al. Determining which patients require evaluation for blunt cardiac injury following blunt chest trauma. World J Surg 2001;25:108-11.

15 Sutherland GR, Driedger AA, Holliday RL, et al. Frequency of myocardial injury after blunt chest trauma as evaluated by radionuclide angiography. Am J Cardiol 1983;52:1099-103.

16 Malangoni MA, McHenry CR, Jacobs DG. Outcome of serious blunt cardiac trauma. Surgery 1994;116:628-33.

17 Schamp DJ, Plotnick GD, Croteau D, et al. Clinical significance of radionuclide angiographically-determined abnormalities following acute blunt chest trauma. Am Heart J 1988;1 16:500-4.

18 Zinck SE, Primack SL. Radiographic and CT findings in blunt chest trauma. J Thorac Imaging 2000;15:87-96.

19 Omert L, Yeaney WW, Protetch J. Efficacy of thoracic computerized tomography in blunt chest trauma. Am Surg 2001;67:660-4.

20 Banning AP, Pillai R. Non-penetrating cardiac and aortic trauma. Heart 1997;17:226-9.

21 Gavelli G, Canini R, Bertaccini P et al. Traumatic injuries: imaging of thoracic injuries. Eur Radiol 2002;12:1273-94.

22 Sakka SG, Huettemann E, Giebe W, et al. Late cardiac arrhythmias after blunt chest trauma. Intensive Care Med 2000;26:792-5.

23 St Louis P, Gandhi S. Cardiac contusion and creatine jinase-MB: a pertinent case history and brief review of the utility of CK-MB. Clin Biochem 1994;27:105-11. 
24 Keller KD, Shatney $\mathrm{CH}$. Creatine phosphokinase-MB assays in patients with suspected myocardial contusion: diagnostic test or test of diagnosis. J Trauma 1988;28:58-63

25 Biffl WL, Moore FA, Moore EE, et al. Cardiac enzymes are irrelevant in the patient with suspected myocardial contusion. Am J Surg 1994:168:523-7.

26 Swaanenburg JC, Klaase JM, DeJongste $M$, et al. Troponin I, troponin $\mathrm{T}, \mathrm{CKMB}$-activity and CKMB-mass as markers for the detection of myocardial contusion in patients who experienced blunt trauma. Clin Chem Acta 1998:272:171-81.

27 King RM, Mucha P Jr, Seward JB, et al. Cardiac contusion: a new diagnostic approach utilizing two-dimensional echocardiography. J Trauma 1983;23:610-4.

28 Walsh P, Marks G, Aranguri C, et al. Use of V4R in patients who sustain blunt chest trauma. J Trauma 2001:51:60-3.

29 Shapiro MJ, Yanofsky SD, Trapp J, et al. Cardiovascular evaluation in blunt thoracic trauma using transesophageal echocardiograph (TEE) J Trauma 1991;31:835-40.

30 Oh JK, Seward JB, Khandheria BK, et al. Transesophageal echocardiography in critically ill patients. Am J Cardio 1990;66:1492-5.

31 Sutherland GR, Calvin JE, Driedger AA, et al. Anatomic and cardiopulmonary responses to trauma with associated blunt chest injury. J Trauma 1981;21:1-12.

32 Sutherland GR, Cheung HW, Holliday RL, et al. Hemodynamic adaptation to acute myocardial contusion complicating blunt chest injury. Am J Cardiol 1986;57:291-7

33 Waxman K, Soliman MH, Braunstein P, et al. Diagnosis of traumatic cardiac contusion. Arch Surg 1986;121:689-92.

34 Holness R, Waxman K. Diagnosis of traumatic cardiac contusion utilizing single photon-emission computed tomography. Crit Care Med 1990; 18:1-4.
35 Godbe D, Waxman K, Wang FW, et al. Diagnosis of myocardial contusion; quantitative analysis of single photon emission computed tomographic scans. Arch Surg 1992;127:888-92.

36 McCarthy MC, Pavlina PM, Evans DK, et al. The value of SPECT-thallium scanning in screening for myocardial contusion. Cardiovasc Intervent Radiol 1991;14:238-40.

37 Lopez-Majano V, Sansi P, Colter R. Nuclear medicine in the diagnosis of cardiac contusion. Eur J Nucl Med 1985;11:290-4.

38 Potkin RT, Werner JA, Trobaugh GB, et al. Evaluation of noninvasive tests of cardiac damage in suspected cardiac contusion. Circulation 1982;66:627-31

39 Iskandrian $\mathrm{AE}$, van de Wall EE, eds. Myocardial viability, 2nd rev ed. Dordrecht: Kluwer, 2000.

40 Chiu WC, D'Amelio LF, Hammond JS. Sternal fractures in blunt chest trauma: a practical algorithm for management. Am J Emerg Med 1997; 15:252-5.

41 Wiener Y, Achildiev B, Karni T, et al. Echocardiography in sternal fracture. Am J Emerg Med 2001;19:403-5.

42 Bu'Lock FA, Prothero A, Shaw C, et al. Cardiac involvement in seatbelt-related and direct sternal trauma: a prospective study and management implications. Eur Heart J 1994;15:1621-7.

43 End A, Rodler S, Oturanlar D, et al. Elective surgery for blunt cardiac trauma. J trauma 1994;37:798-802.

44 Stone DL, Fleming HA. Aneurysm of the left ventricle and left coronary artery after non-penetrating chest trauma. Br Heart J 1983;50:495-7.

45 Grieco JG, Montoya A, Sullivan HJ, et al. Ventricular aneurysm due to blunt chest injury. Ann Thorac Surg 1989;47:322-9.

46 Sybrandy KC, Oosterom L, Burgersdijk C, et al. An unexplained right ventricle dilatation clarified by a cardiac contusion many years previously. Ned Tijdschr Geneesk 2001;145:2443-6.

\section{ELECTRONIC PAGES}

\section{eHEART: www.heartinl.com}

j he following electronic only articles are published in conjunction with this issue of Heart.

\section{Williams syndrome associated with complete atrioventricular septal defect}

S Nakamoto, T Saga, T Shinohara

Williams syndrome is a genetic disorder associated with characteristic facies, supravalvar aortic stenosis, peripheral pulmonary stenosis, mental retardation, hypertension, premature aging of skin, and congenital cardiac defects. Many cardiac defects such as bicuspid aortic valve, mitral valve regurgitation, coarctation of the aorta, and ventricular or atrial septal defects are linked to the syndrome. Complete atrioventricular septal defect has rarely been associated with Williams syndrome and only one necropsy case has been reported in the literature. The long term follow up of Williams syndrome associated with complete atrioventricular septal defect is reported. During a 10 year follow up period, the pressure gradient in the ascending aorta did not increase despite narrowing of the ascending aorta as identified on an aortogram.

(Heart 2003;89:el5) www.heartjnl.com/cgi/content/full/89/ $5 / \mathrm{e} 15$

\section{Innocent victim of a localised outbreak: legionella} endocarditis

R Massey, P Kumar, J R Pepper

Legionella pneumophila endocarditis is extremely rare. The case of a fit 26 year old man who had previously undergone homograft aortic root replacement is reported. He was admitted with legionella pneumonia during the recent localised outbreak but went on to develop endocarditis. His aortic valve was replaced with a mechanical valve and he made an uneventful recovery. Public health issues and diagnosis in susceptible patients during localised outbreaks are discussed.

(Heart 2003;89:el6) www.heartjnl.com/cgi/content/full/89/ $5 / \mathrm{e} 16$

\section{Trash feet after coronary angiography}

A M Khan, S Jacobs

Cholesterol crystal embolisation is a frequently underdiagnosed condition. While coronary catheterisation is safe and commonly performed, the reported patient developed very painful trash feet after undergoing this routine procedure. Ulceration and gangrene occurred after catheter manipulation during cardiac angiography, which caused occlusion of the small arteries in his feet. The triad of pain, livedo reticularis, and intact peripheral pulses is pathognomonic for cholesterol embolisation. The prognosis depends on the extent of the systemic disease and a high rate of mortality $(75-80 \%)$ is observed. Prognosis is poor and the treatment is only supportive. It is suggested that while cardiac catheterisation is largely safe and a very commonly performed procedure, it can still lead to complications with serious side effects and can even prove fatal.

(Heart 2003;89:el7) www.heartjnl.com/cgi/content/full/89/ $5 / \mathrm{e} 17$ 